\title{
Visible Light Communication [VLC] and its Applications
}

\author{
Dr. Asha T $\mathbf{S}^{1}$, Drisya $\mathbf{K}^{2}$ \\ Professor, Electronics and Communication Engineering, NSS College of Engineering, Palakkad, India ${ }^{1}$ \\ PG Student, Communication Engineering, NSS College of Engineering, Palakkad, India ${ }^{2}$
}

\begin{abstract}
The communication using visible light is the new trend in communication field where the different coloured light or different intensity of light is used to communicate. Why the visible light communication is more important in this era? It is because the already existing communication method has some difficulties and interference. The most of the existing communication systems uses radio frequency. In radio communication, information's such as sound, images are carried by radio waves. But this Radio Frequency [RF] communication has some disadvantages. It suffers from interference and high latency issues and the RF communication requires a separate setup for transmission and reception of radio waves. For overcoming these limitations, a new method is adopted ie, Visible Light Communication [VLC].
\end{abstract}

Keywords: Visible Light Communication, Optical Wireless Communication, Li-Fi, Light Emitting Diode, Laser Diode

\section{INTRODUCTION}

The radio frequencies occurs in the range of $30 \mathrm{HZ}$ to $300 \mathrm{GZ}$. But only a small part of this spectrum is used for commercial applications. A radio communication system sends signals by radio. Types of radio communication systems deployed depend on technology, standards, regulations, radio spectrum allocation, user requirements, service positioning and investment. The radio frequency communication have noticeable advantages over all other aids of communication and used in huge amount of applications. Radio communication systems can transmit large amount of information into distant destinations. It have relatively low construction costs compared with other forms of communication systems since it does not uses physical cable and expensive attenuation equipment. Attenuation equipment is the device which maintain signal strength during transmission.

Now the mobile data traffic is increasing drastically. This increase is due to the increase in number of devices accessing the mobile network. Further, the online social services such as Facebook, WhatsApp and Twitter also increases the mobile data traffic. Beyond this, interference is the another important issue in radio communication. According to Federal Aviation Administration [FAA], the use of mobile phones on aircraft causes interference with communication and navigational systems. The Federal Communication Commission [FCC] states that the mobile phones on aircraft will cause interference with ground system towers[1]. One of the major need of any wireless communication system is that it should have very low latency. There is also security issues incase of radio frequency communication techniques due to the penetration of waves through walls. The increase in the transmission power of RF waves beyond a certain limit results in risks to human health. There is also power inefficiencies incase of RF communication due to the requirement of a separate setup for communication of the RF waves.

Due to the disadvantages of RF communication, a new method of communication has been invented and is

known as Visible Light Communication[VLC]. Visible light communication system uses visible light for communication. Visible light communication is a data communications variant which uses visible light between 400 800 THZ frequency [780-375 nm wavelength]. VLC can be used as a communication medium for ubiquitous computing because light producing devices such as indoor/outdoor lamps, TVs, traffic signs, commercial displays and car headlights/taillights are used everywhere. Using visible light is also less dangerous for high power applications because human can perceive it and act to protect their eyes from damage.

The history of visible light communication have a journey of several years. In ancient times, fire and smoke signals are used to convey messages. In Roman, the long distance communication is possible by sunlight reflection from polished metallic plates. During 1794, Claude Chappe in France developed the "semaphore" means of visual signaling. This is accomplished by means of flags or different coloured lights. The first visual telegraphy system was also developed by Claude Chappe. After that, in the early 1800s, a wireless solar telegraph, known as Heliograph was developed by the US military. It was based on Morse code flashes of reflected sunlight by a mirror. In 1880s in Washington, D.C. When the Scottish scientist Alexander Graham Bell invented the photophone, which transmitted speech on modulated sunlight over several hundred meters. It was a huge change from transmission of speech by radio to transmission of information by visible light. Optical communication technologies did not attain much popularity till the development of Light 


\title{
International Advanced Research Journal in Science, Engineering and Technology
}

\author{
Vol. 6, Issue 2, February 2019
}

Amplification by Stimulated Emission of Radiation [LASER]. During 1970, the optical fibers with commercial applications has been successfully developed and which have low attenuation. At the same time, the GaAs semiconductor laser for use in optical fiber cables for long distance communication was also developed. More recent work began in 2003 at Nakagawa Laboratory, in Keio University, Japan using LEDs to transmit data by visible light. After that there have been numerous research activities focused on VLC. There after, in 2006, researchers from CICTR at Penn State proposed a combination of Power Line Communication [PLC] and white light LED to provide broadband access for indoor applications[2]. This research suggested that VLC could be deployed as a perfect last-mile solution in the future. In January 2010 a team of researchers from Siemens and Fraunhofer Institute for Telecommunications, Heinrich Hertz Institute in Berlin demonstrated transmission at 500Mbit/s with a white LED over a distance of 5 metres and 100Mbit/s over longer distance using five LEDs[3]. The VLC standardization process is conducted within IEEE Wireless Personal Area Networks working group[802.15].

In December 2010 St.Cloud, Minnesota, signed a contract with LVX Minnesota and became the first to commercially deploy this technology[4]. The LVX system is the world's most innovative and pioneering utility. They are the first to patent and offer a super high-quality LED light. Such LED light securely transmit high-speed data. In July 2011, TED Global had succeeded in the live demonstration of the transmission of high-definition video using a standard LED lamp[5]. Recently, VLC-based indoor positioning systems have become an attractive topic. ABI research forecasts that it could be a key solution to unlocking the $\$ 5$ billion "indoor location market"[6]. Publications have been coming from Nakagawa Laboratory ByteLight filed a patent on a light positioning system using LED digital pulse recognition in March 2012 COWA at Penn state and other researchers around the world.

Another recent application of VLC is in the world of toys. The manufacturing of toys becomes cost efficient and low complex. The implementation requires one micro-controller and one LED. Recently Organic LEDs [OLED] have been used as optical transceivers to buildup VLC communication links up to 10Mbit/s[7]. In October 2014, Axrtek launched a commercial bidirectional RGB LED VLC system called MOMO that transmits down and up at speeds of 300Mbit/s and with a range of 25 feet[8]. In May 2015, it is established the VLC location based services to customers smartphones in a hypermarket in Lille, France. In June 2015, two Chinese companies, Kuang-Chi and Ping An Bank, partnered to introduce a payment card that communicates information through a unique visible light. In March 2017, Philips set up the first location-based services to customers smartphones in Germany. One of the major disadvantage of RF technology, ie spectrum congestion is removed by using visible light spectrum, where ultra wide range of unregulated spectrum is available.

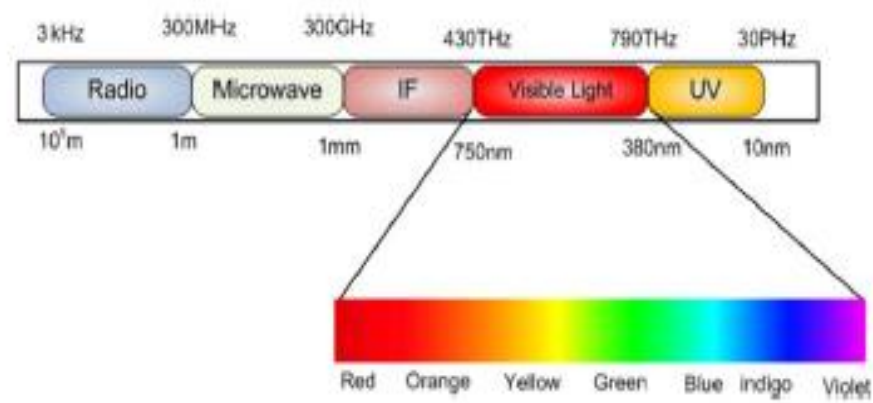

Fig1. VLC Frequency Spectrum [1]

\section{A. Optical Wireless Communication [OWC]}

The optical part of the electromagnetic spectrum is considered as a strong solution for the future high density, high capacity networks. The method of establishing wireless connectivity by means of optical spectrum is known as Optical Wireless Communication[OWC]. The coexistence of OWC and RF systems may provide an effective solution for the huge demand of upcoming $5 \mathrm{G}$ and more advanced communication systems. OWC refers to the optical transmission in the guided Visible Light[VL], infrared[IR] and ultraviolet[UV] spectrum. OWC systems operating in the visible light band are commonly known as Visible Light Communication [VLC]. Different OWC technologies include VLC, Light Fidelity [LiFi], Optical Camera Communication[OCC], FSO Communication[FSOC] and Light Detection And Ranging[LiDAR]. The OWC application areas include industry, health care, railway stations, transportation, homes, offices, shopping malls, underwater and space. For these application platforms, all the type of communication like device-to-device[D2D], machine-to-machine[M2M], chip-to-chip, device/machine, device/machine-to-user, user-todevice/machine, vehicle-to-infrastructure, vehicle-to-vehicle, infrastructure-to vehicle[V2X], point-to-point, multipointto-point, point-to-multipoint are used. The OWC systems provide high data rate services. The communication distance can range from a few nanometers to more than $10,000 \mathrm{~km}$ and it is suited for both indoor and outdoor services. The major disadvantage of such systems are sensitivity to blocking by obstacles and limited transmission power[9]. 


\section{International Advanced Research Journal in Science, Engineering and Technology}

Vol. 6, Issue 2, February 2019

\section{B. Architecture of VLC}

All VLC systems consists of two common parts namely the transmitter and the receiver. These two parts generally have three layers, namely physical layer, MAC layer and application layer. The layered architecture of VLC system is shown in fig 2[10]. In IEEE 802.15.7, only two layers [such as PHY and MAC] are defined for simplicity[11].

1) MAC layer: The tasks performed by Medium Access Control [MAC] layer include[12]

- $\quad$ Mobility support

- $\quad$ Dimming support

- $\quad$ Visibility support

- Security support

- $\quad$ Schemes for mitigation of flickering

- $\quad$ Colour function support

- Network beacons generation if the device is a coordinator

- VPAN disassociation and association support

- $\quad$ Providing a reliable link between peer MAC entities

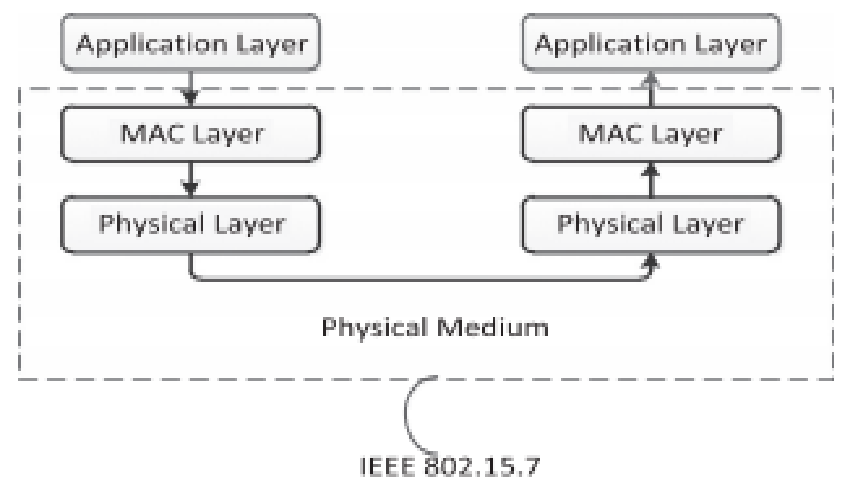

Fig 2. Layered architecture of VLC [1]

The MAC layer support topologies like peer-to-peer, broadcast and star.

2) Physical layer: The main functions of physical layer includes providing physical specification of the device and the relationship between the device and the medium. Fig 3. shows the typical physical layer system model of VLC. First the input bit stream is passed through a channel encoder. The most commonly used channel encoders are linear block codes, convolutional codes and turbo codes. The channel encoded bit stream is then passed through a line encoder to produce a line encoded bit stream. Then it passes through a modulation module. The most commonly used modulation techniques include ON-OFF Keying, PPM and PWM. Finally, the data is fed to the LED or LD for transmission through the optical channel. The bidirectional transmission of VLC system utilizes Wavelength Division Multiplexing[WDM] and SubCarrier Multiplexing [SCM]. The data rate can be increased by Orthogonal Frequency Division Multiplexing[OFDM] and Quadrature Amplitude Modulation[QAM][13].

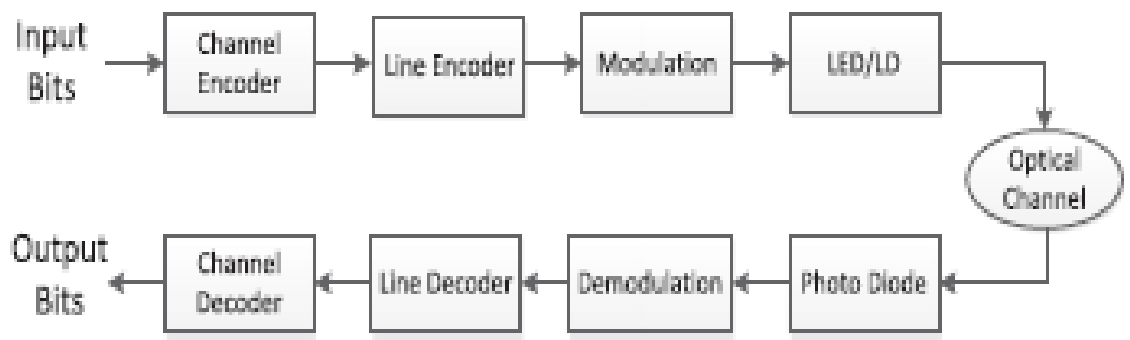

Fig 3. Typical physical layer system model of VLC [1] 


\section{International Advanced Research Journal in Science, Engineering and Technology}

Vol. 6, Issue 2, February 2019

\section{VLC SYSTEM IMPLEMENTATION}

The typical VLC system can be implemented using diodes and sensors. Typical transmitters used for visible light communication are visible light LEDs and receivers are photodiodes and image sensors. LED[Light Emitting Diodes] are the latest and most exciting technological advancement in the lighting industry. The LEDs are small, solid light bulbs which are extremely energy efficient and long lasting. Now, the white LEDs are used as the efficient light sources for communication and illumination and are replacing existing incandescent light bulbs[14]. Fig 4. shows the LED luminous efficiency improvement curve. From the curve, it can be concluded that the efficiency of LED lamps and luminaires are increasing year by year. The current LED lamps typically have a lifetime of 40,000 hours, and this is 40 times more than incandescent lamps. Fig 5. shows the trend existing between price of LED light and improvement in LED technology.

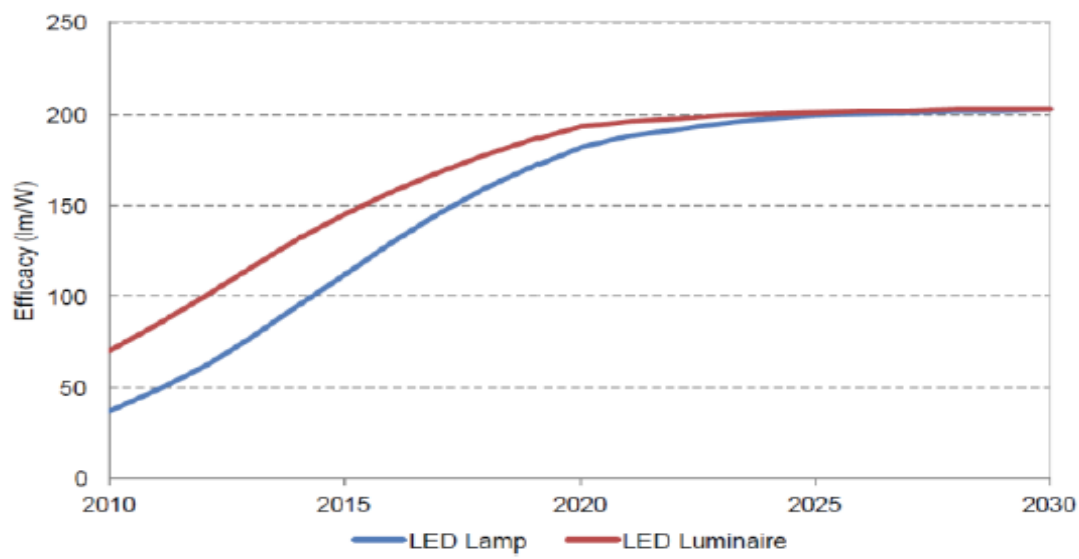

Fig 4. LED Luminous Efficiency Improvement [14]

When the LED light is used for illumination, its brightness has to be controlled. The commonly used receivers for visble light communication includes photodiodes and sensors. An image sensor is able to do image acquisition and data reception simultaneously. Such a sensor continuously takes images of a scene using an LED light. The intensity of the LED light is modulated and the receiver detect the intensity from the pixel where the light is focused on. The advantage of image sensor over a single photodiode is that, since we are using visible light as communication medium, the occurrence of interference is sound in all the conditions. The interfering light is focussed on all the pixels except the pixel in which the desired signal is focussed. This shows that image sensor reception is much more robust against interference as compared to single photodiode reception. The image sensors in visible light communication can also be used for accurate position detection[14].

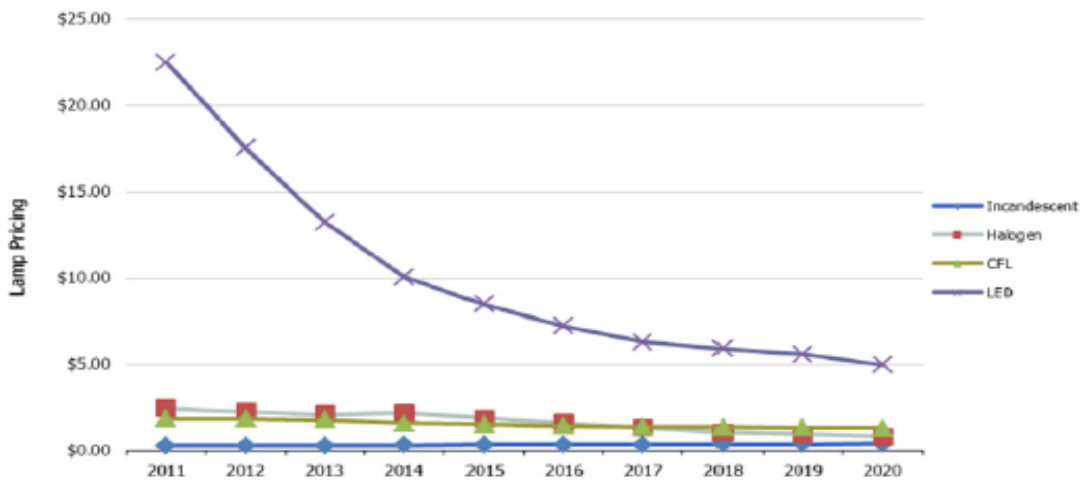

Fig 5. 60 Watt LED light price trend [14]

As compared to other short range wireless technologies such as IrDA and bluetooth, the LED communication has some limitations. It has limited range of communication and a much slower data rate. But over these limitations, what make LED communication advantageous is that, it can be implemented at a fraction of the cost and in some situations, it may be free. This is because LED communication is essentially a software interface technique using existing hardware and the required modification is less[15]. The LED communication techniques are commonly implemented in situations where traditional techniques are much more expensive. 


\title{
International Advanced Research Journal in Science, Engineering and Technology
}

\author{
Vol. 6, Issue 2, February 2019
}

In wireless mobile communications, the size of the cell is constantly reducing over the last decades and now the transmission within pico- or femto-cells is being proposed[16]. In such a condition, the optical wireless communication becomes an attractive alternative to radio links. In this case, LED is used as the light source and the receiver side is completed using avalanche or p-i-n photodiodes. These solution allow transmission in excess of 1Gbit/s[16]. The most important characterization of the LED is the L-I [Light intensity-Current] characteristic. The measurement was carried out using an ILX Lightwave current source to drive the LED and a power metre to measure the LED output power. The output parameter is recorded in intervals of 10mA and these results were graphed with error fit as shown in fig 6[17]. The white LED is considered as the major lighting for the next generation. This advantage is because, compared with conventional lighting, white LEDs have lower power consumption, lower voltage, longer lifetime, smaller size and cooler operation. [18] proposed a wireless optical communication system that uses white LEDs for indoor wireless networks. The medium used is visible light from LED lighting. The advantages of this system includes:

- $\quad$ The high power lighting equipment is used to enable communication throughout the whole room.

- $\quad$ Lighting equipment with white coloured LEDs is easy to install and nice looking.

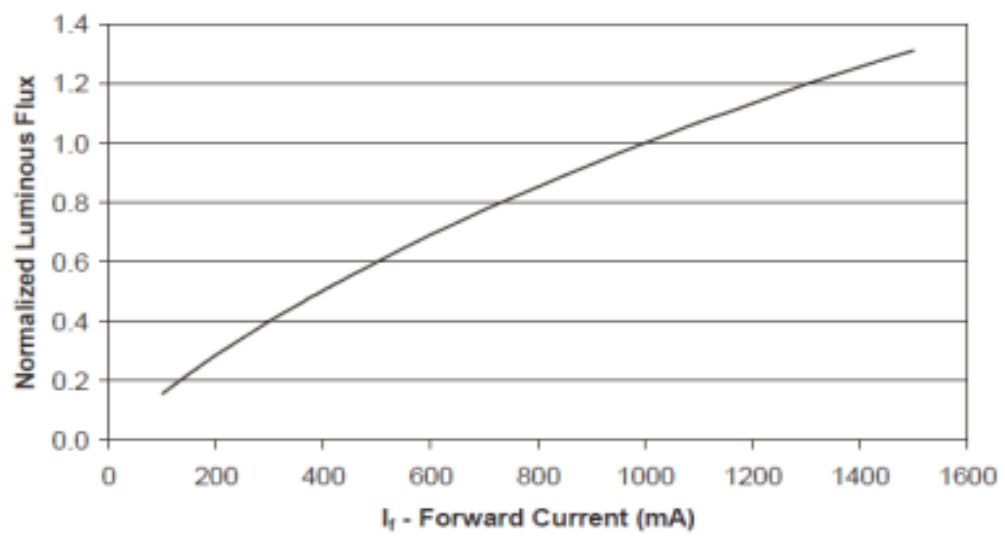

Fig 6. L-I characteristic of source LED [17]

An LED can able to emit and receive light and a microcontroller can be used to provide synchronization. By providing synchronization, the receiver can lock to the transmitted signal in a fast and efficient way. An LED can be used to emit as well as receive light at the same time by multiplexing approach. The LED can be used to achieve bidirectional communication and in such a condition, the use of photodetectors can be neglected. Bidirectional communication is obtained by temporal separation of transmitter and receiver signals. Such a system can be finished by using single LEDs for transmission and reception using an On-Off Keying modulation. The resultant system is a low-power one for low data rate applications[19].

Analog photonic link is a new idea in the communication area in which radio frequency signals are transmitted through optical fiber. This is a promising technology used in antenna remoting, radio-over-fiber and phased-array radar systems and fiber provides benefits in bandwidth, transmission loss, immunity to electromagnetic interference and reduced size and weight as compared to coaxial cable[20]. The key figures-of-merit of an analog photonic link includes increased gain, lower the noise figure[NF], improve the spurious free dynamic range[SFDR]. Generally the visible light communication using LED is possible through IM/DD links. The modification to optical signals, ie phase modulated optical signals can be generated more efficiently and are less susceptible to fiber nonlinearity compared to normal intensity modulated signals. The phase modulated analog photonic link has a larger peak gain and a lower noise figure compared to the conventional IM/DD link[21]. Another advantage is that the phase modulated analog photonic link does not require a bias control circuit for the phase modulator at the transmitter, but at the receiver. High-power and high-linearity photodiodes are used to improve the figure-of-merit of the analog photonic link because the figures-ofmerit of link generally improve with higher photocurrent.

\section{MODULATION SCHEMES FOR VLC}

By enabling recent advances in LED technology, IEEE 802.15.7 supports high-data-rate visible light communication upto $96 \mathrm{Mb} / \mathrm{s}$ by fast modulation of optical light sources. IEEE 802.15.7 provides dimming adaptable mechanisms for flicker-free high-data-rate visible light communication. The two main challenges for communication using visible light spectrum are flicker mitigation and dimming support. The fluctuation in light brightness is known as flicker. To avoid flicker, the changes in brightness must fall within the Maximum Flickering Time Period[MFTP]. The MFTP is defined 


\title{
International Advanced Research Journal in Science, Engineering and Technology
}

\author{
Vol. 6, Issue 2, February 2019
}

as the maximum time period over which the light intensity can change without the human eye perceiving it. There is no generally accepted optimal flicker frequency number, a frequency greater than 200HZ[MFTP $<5 \mathrm{~ms}$ ] is generally considered as safe. Another important factor for VLC is dimming support. This gives the advantages of power saving and energy efficiency. The human eye's response is limited to low light levels by enlarging the pupil, thus more light rays are enter into the eye. This response results in a difference between perceived and measured levels of light.

The relation between these two parameters are given by[22],

$$
\text { Perceived light }[\%]=100 \times \sqrt{(\text { measured light } \div 100)}
$$

The existing national trade organizations like Japan Electronics and Information Technology Industries Association[JEITA], Visible Light Communications Consortium[VLCC] and Home Gigabit Access Project[OMEGA] in Europe does not focus on flicker mitigation and dimming support. The IEEE 802.15.7 mainly concentrated on this parameters. The IEEE 802.15.7 standard supports multiple diverse topologies, such as peer-to-peer and star topologies with data rates ranging from $11.67 \mathrm{~kb} / \mathrm{s}$ to $96 \mathrm{Mb} / \mathrm{s}$ for indoor and outdoor applications.

\section{A. Modulation Methods in 802.15.7}

The IEEE 802.15.7 standard offers three physical[PHY] layer types for VLC. PHY I operates from 11.67 to 266.6kb/s, PHY II operates from 1.25 to $96 \mathrm{Mb} / \mathrm{s}$ and PHY III operates between 12 and 96Mb/s. PHY I and PHY II are defined for a single light source, and support On-Off Keying[OOK] and Variable Pulse Position Modulation[VPPM]. Phy III uses multiple optical sources with different frequencies[colours] and use a particular modulation format called Colour Shift Keying[CSK]. The most important modulation scheme used for VLC includes OOK modulation, in which the LEDs are turned on or off dependent on the data bits being send[either 1 or 0 ].

\section{B. Dimming Mechanisms for Flicker-Free Communication}

1) Idle Pattern Dimming: An idle pattern is defined as a pattern whose duty cycle variation results in a change of brightness to support dimming. The idle pattern dimming maintain visibility and flicker-free operation.

2) Visibility Pattern Dimming: Visibility pattern dimming is similar to idle pattern dimming except that the patterns are used inside the payload visibility frame. The features such as flicker mitigation, continuous visibility, device discovery and colour stabilization are supported by visibility pattern.

3) Flicker Mitigation: According to the generation mechanism, the flicker in VLC can be classified into two categories.

- Intra-frame flicker

- Inter-frame flicker

Intra-Frame Flicker Mitigation: Intra-frame flicker is defined as the perceivable brightness fluctuation within a frame. Intra-frame flicker mitigation refers to mitigation of flicker within the transmission of a data frame.

Inter-Frame Flicker Mitigation: Inter-frame flicker is defined as the perceivable brightness fluctuation between adjacent frame transmissions. Inter-frame flicker mitigation applies to both data transmission and idle periods.

In [23], the Non-Orthogonal Multiple Access[NOMA] technique to improve the achievable sum rate of multiple input multiple-output[MIMO] based multi-user visible light communication[VLC] systems were proposed. In practical VLC systems, there is only one LED transmitter and it is expected to support large number of receivers, ie multiple access is the method used in multi-user VLC systems. Orthogonal Frequency Division Multiplexing[OFDM] is a method of encoding digital data on multiple carrier frequencies. Orthogonal Frequency Division Multiple Access[OFDMA] is a multi-user version of the popular OFDM digital modulation scheme. Multiple access is achieved in OFDMA by assigning subsets of subcarriers to individual users. In NOMA systems, all users can use the entire modulation bandwidth of the system through power domain superposition coding at the transmitter side and Successive Interference Cancellation[SIC] at the receiver side[23].

A new trend in Visible Light Communication, ie communication using LED and mobile phone cameras is attractive and it provides low-cost wireless communication. [24] proposes a VLC system using Colour Shift Keying[CSK] modulation and Code Division Multiple Access[CDMA]. The use of image sensors or cameras as VLC receiver has several advantages. The new generation mobile phones equipped with embedded Complementary Metal-OxideSemiconductor[CMOS] cameras allowing users to capture photos and videos. CDMA is the important technology that provide multiple users to access the network simultaneously. CSK is used to enhance the VLC system capacity while CDMA allows multiple users to access the network. CSK scheme enhanced the system throughput and avoid the single colour interference. While CDMA allowed multiple users to access to the network. OFDM is widely used in VLC 


\section{International Advanced Research Journal in Science, Engineering and Technology}

Vol. 6, Issue 2, February 2019

systems due to its advantages. The Optical OFDM[O-OFDM] signals are used to activate the LEDs. There are two methods existing for realising O-OFDM signals.

- $\quad$ Direct Current Biased O-OFDM[DCO-OFDM]

- $\quad$ Asymmetrically Clipped O-OFDM[ACO-OFDM]

DSO-OFDM converts complex input signals into real values and then adds a DC bias to change the resultant signals to strictly unipolar ones. In ACO-OFDM, the real/unipolar OFDM samples are obtained by only modulating the odd subcarriers and then clipping all negative values at the zero level. The general OFDM signal has high peak-to-average power ratio[PAPR] due to multicarrier modulation. This high PAPR results in severe non-linear distortion because of the LED non-linearities in VLC systems. By DCO-OFDM, large DC bias is needed for high signal peak and worse the non-linear distortion.

\section{LIFI: THE FUTURE TECHNOLOGY IN WIRELESS COMMUNICATION}

Light Fidelity[LiFi] is one of the future technology in wireless communication area. LiFi is similar to Wireless Fidelity[WiFi]. LiFi is bidirectional communication with very high speed and is a fully networked communication. In this technology, the information is transmitted by varying the intensity of the light. This variation is faster than human eye can capture. The data transmission in LiFi uses LED bulbs with transceiver and it is about 100 times faster than WiFi. LiFi technology was proposed by the German scientist Harald Haas.

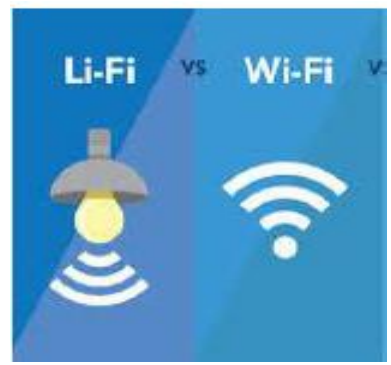

Fig 7. Symbol of LiFi and WiFi [24]

The advantages of LiFi includes:

- Higher bandwidth

- $\quad$ Efficiency

- $\quad$ Availability

- Security

- High speed

LiFi uses the license free light spectrum and the communication is possible through use of LED bulbs. The visible light communication spectrum has no side effects. In this spectrum 10,000 times more space is available. LiFi is the fast and cheap optical version of $\mathrm{WiFi}[24]$.

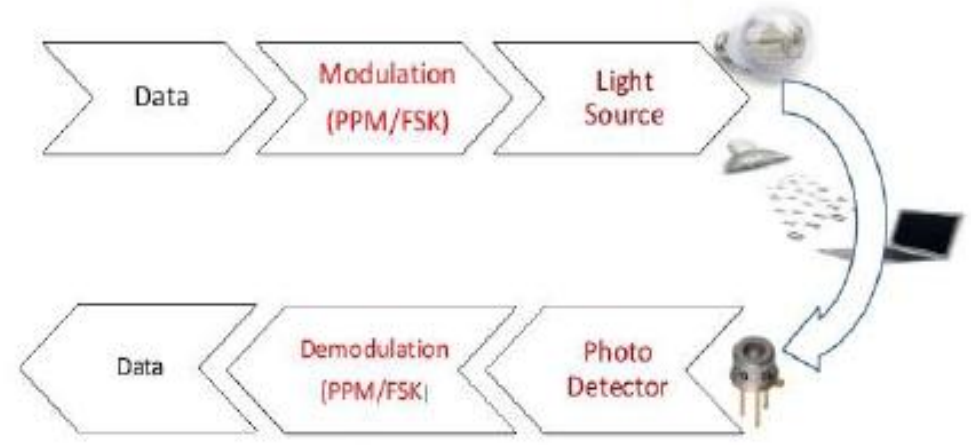

Fig 8. Block diagram of LiFi Technology [24]

The applications of LiFi includes LiFi for desktops, smartcard LiFi, LiFi for schools and hospitals, LiFi in cities, smart guides, museums, hotels, fairgrounds, eventsindoor and LBS[Location Based Services], access control and 


\section{International Advanced Research Journal in Science, Engineering and Technology}

Vol. 6, Issue 2, February 2019

identification crisis, malls, airport and dangerous environments like thermal power plants. The table 1 below shows the comparison between LiFi and WiFi technology.

\section{A. Advantages of LiFi [26]}

- It supports the user with more than sufficient communication speed for downloading movies, music, games and all in very less time.

- $\quad$ Capacity : Light has 10,000 times wider bandwidth than radio waves

- $\quad$ Efficiency : Since the LED light consumes only very little energy, the LiFi technology is highly efficient.

- Availability: The transmitters and receivers for this communication is available locally.

- $\quad$ Security : Light waves are more secure than electric waves because they cannot penetrate through walls.

- Utilization : The use of LiFi technology is mainly concentrated in the areas where WiFi cannot be used such as under water, aircraft cabins and nuclear power plant.

- $\quad$ Safety : Light communication methods like LiFi are safe as compared to radio means of communication because $\mathrm{LiFi}$ is not able to penetrate through the body.

Table 1.Comparison between LiSSSFi and WiFi [24]

\begin{tabular}{|l|l|l|}
\hline Parameters & LiFi [Light Fidelity] & WiFi [Wireless Fidelity] \\
\hline Range & $\begin{array}{l}10,000 \text { times broader } \\
\text { than that of RF }\end{array}$ & $\begin{array}{l}\text { much lesser than the } \\
\text { spectrum range of VL }\end{array}$ \\
\hline Speed & High & High \\
\hline Data density & High & Low \\
\hline Security & High & Medium \\
\hline Reliability & Medium & Medium \\
\hline Power available & High & Low \\
\hline Transmit/receive power & High & Medium \\
\hline Ecological impact & Low & Medium \\
\hline $\begin{array}{l}\text { Device-to-device } \\
\text { connectivity }\end{array}$ & High & High \\
\hline Obstacle interference & High & Low \\
\hline Cost & $\begin{array}{l}\text { Cheaper than WiFi } \\
\text { because it uses light }\end{array}$ & $\begin{array}{l}\text { Costlier than LiFi because } \\
\text { it uses radio spectrum }\end{array}$ \\
\hline Market maturity & Low & High \\
\hline
\end{tabular}

\section{B. Limitations of LiFi [26]}

- Interference from natural light sources present within the communication medium.

- The system works only if there is a line-of-sight between transmitter and receiver.

- Reliability issues

- $\quad$ Coverage area

\section{Applications of LiFi [26]}

- Medical Applications: Due to the radiation problems associated with the WiFi technology, LiFi is the appropriate technology that can be used in hospitals. The radio signals cause interference with various monitoring equipments present in the hospitals like MRI equipment. The LiFi technology is beneficial for robotic surgeries.

- $\quad$ Road Safety and Traffic Management: Vehicles can communicates using LiFi through the LED lights.

- $\quad$ Smart Lighting: Any light source, private or public like street lamps can be used for providing LiFi hotspot as well as for lighting. 


\section{International Advanced Research Journal in Science, Engineering and Technology}

Vol. 6, Issue 2, February 2019

- $\quad$ Aviation: Aircraft cabins already have LED lights which can be used to provide high speed internet access.

- Underwater Communication: Radio waves are quickly absorbed in water and acoustic waves disturb marine life. But LiFi technology overcome all such limitations.

Now the LiFi communication using white LEDs are commonly used. The recent trends are focusing on parallel data transmission by using multiple LEDs or array of LEDs. In this case, each LED transmits a different stream of data. The red, blue and green LEDs are considered as different data channels by altering the light frequencies[24]. Every LiFi product primarily consist of Light Emitting Diode[LED], RF power amplifier circuit[PA], printed circuit board[PCB] and enclosure kit[27]. The LiFi technology is based on the

visible light communication and it uses visible light for data communication. In this, we can use a LED lamp as the source of illumination as well as for sending information. Therefore, VLC is illumination along with communication[28].

The headlights and taillights of vehicles can be used for providing road safety and traffic management. This approach is also an application of LiFi technology. In this method, a unique code is assigned to every vehicle. The head and tail lights of the vehicle transmit this code in regular intervals. When the vehicle passes through the toll plaza, the code is send to the PC at toll plaza and the complete information about the vehicle will be displayed on the PC. Traffic police can make use of this information if required. The person then pays the toll [Road Service Tax] and move ahead. Thus information about each and every vehicle passed, number of vehicle passed in one day and the total service tax collected is recorded in the central server of such road service tax system. All the computers at all toll plazas are networked together. Thus they can share the required information about each and every vehicle. The anti nationalists normally use stollen cars for their activities. The normal tendency is to change the number plates and use the cars. But in this system, it will not be possible to do so, it is because, in such cases the signal transmitted by vehicle head and tail light will display the information on the PC at toll plaza but it does not match with its number. Therefore, such vehicles can be stopped immediately[30].

The LiFi technology can be used to provide protection against vehicular collisions on the roads. LEDs are used as the transmitters and which communicate with photosensors located at the receiver side. The white LEDs used in the head and tail lights can effectively used for short range communication with the photodetectors. White LEDs are the suitable solution for indoor communication applications where short range, high speed wireless data transmission is possible. The transmitter and receiver circuits are comparatively inexpensive and the complexity is also less. The transmission upto a range of $0.45 \mathrm{~m}$ can be achieved[31]. The best method to prevent collisions between vehicles on the roads can be done by communication among them.

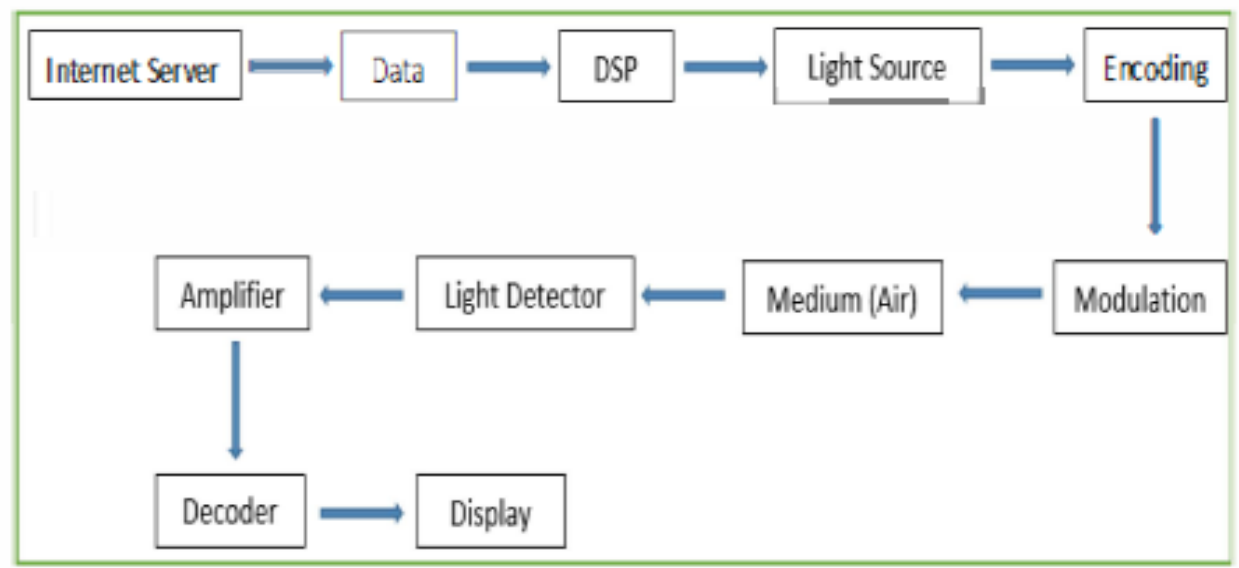

Fig 9. Implementation of smart vehicular communication system [31]

The optical power generated internally by an LED is given by

$$
P_{\text {int }}=R_{r} h \vartheta=n_{\text {int }} \frac{i}{e} h \vartheta
$$

where $R_{r}$ is equivalent to number of photons generated per second. h $\vartheta$ is the energy of each photon, $n_{\text {int }}$ is the internal efficiency, $i$ is the input current and $\mathrm{e}$ is the elementary charge on an electron. The generated power of the LED varies depending on the semiconductor material used for fabrication and depending on the semiconductor material, the input current varies. 


\section{International Advanced Research Journal in Science, Engineering and Technology}

Vol. 6, Issue 2, February 2019

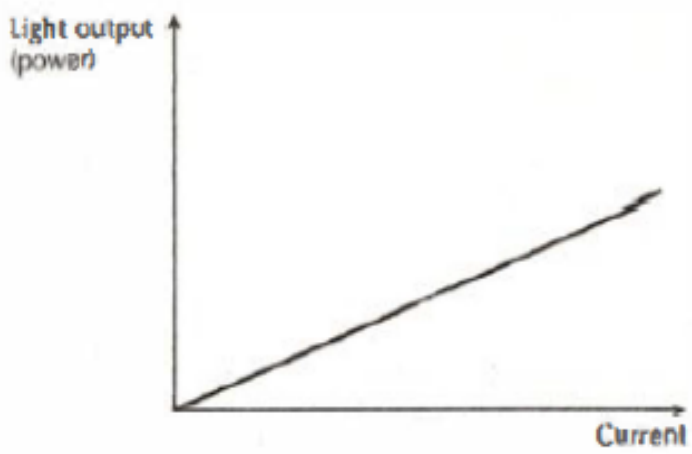

Fig 10. LED output characteristics [31]

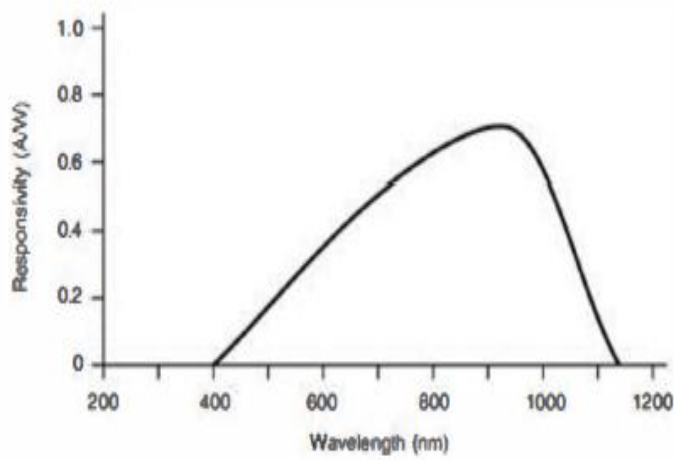

Fig 11. Photodiode Response [31]

The sensitivity to light by a silicon photodiode can be measured by the time response. It is defined as the ratio of photocurrent IP and incident light power at a given wavelength.

$$
R_{\lambda}=\frac{I_{P}}{P}
$$

The responsivity varies with the wavelength of incident light.

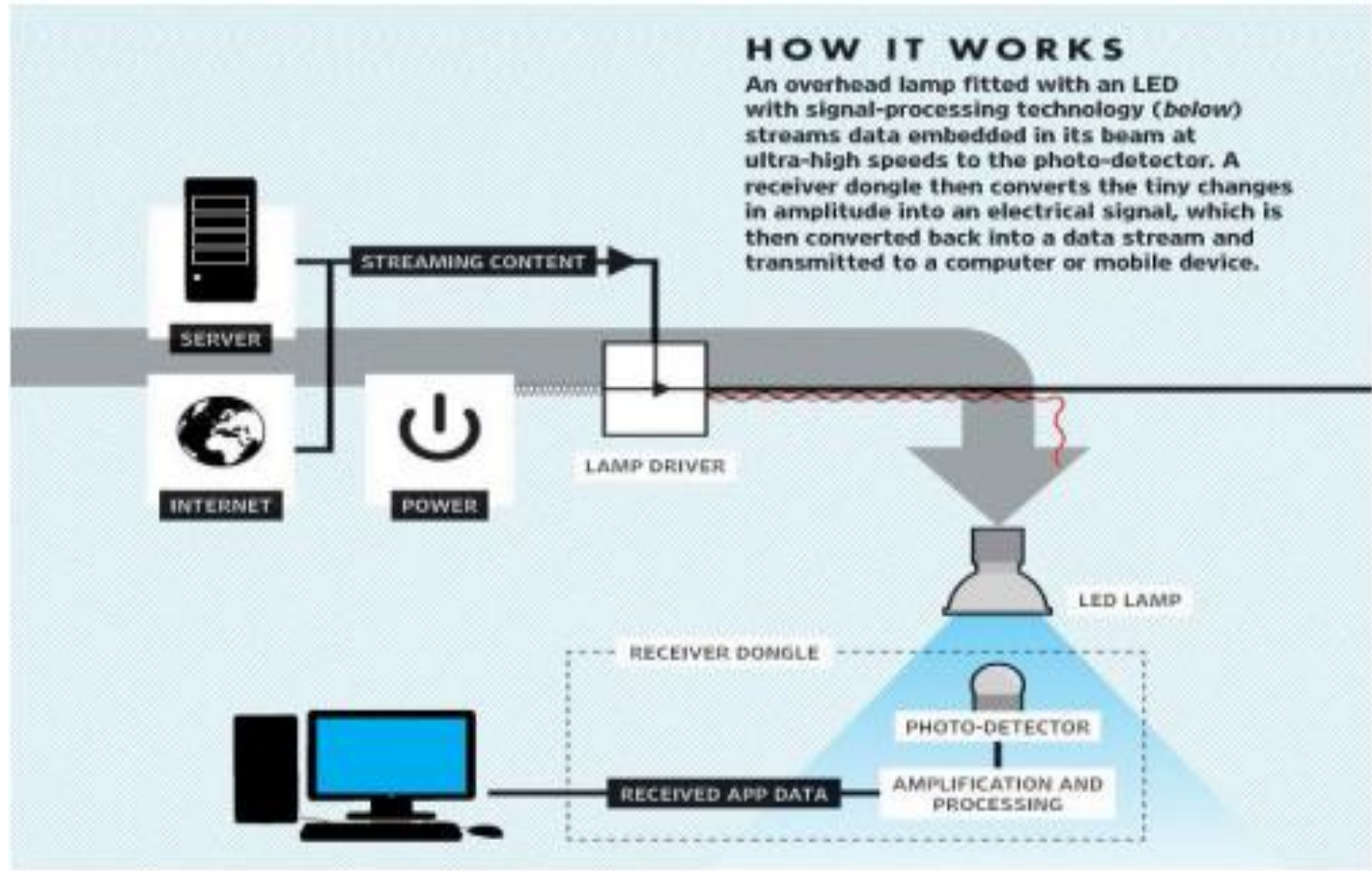

Fig 12.Working mechanism of LiFi [33] 


\title{
International Advanced Research Journal in Science, Engineering and Technology
}

\author{
Vol. 6, Issue 2, February 2019
}

[33] proposed a new method to manage traffic and to maintain the safety of a road using LiFi technology. In this a unique number is assigned to each vehicle and against this number the information is stored in the database. When a car violate the traffic signal or crosses the maximum speed limit, the information along with the unique number of the car goes to the central server through LiFi. The information in the server will helps to take legal actions against the vehicles. Another condition is that, if two vehicles crosses the minimum distance which is necessary to prevent accident, a signal is send to the second vehicle to reduce the speed for avoiding accidents.

The combination of both efficient traffic management methods and proper security measures in vehicle-to-vehicle communication techniques leads to an increase in road safety and prevent vehicle theft. In the proposed system, street light is used as the main medium to transmit and receive information to and from the vehicles present in the road. The data is distributed using Power Line Communication[PLC]. The modulation is performed using LED and the information is sent out as modulated light pulses from street lights. Each vehicle has a receiver module that contains a photodiode. The photodiode converts light in to current and it can be feed to appropriate output devices such as LED or speaker. But there is a limitation due to the LOS feature of LiFi. Due to this limitation, the data cannot be transmitted directly to every car using street lights as there will be a obstruction from intermediate cars[34].

[35] proposes a block diagram for the VLC system. The transmitter section consists of digital to analog converter, transconductance amplifier, low pass filters and high speed LEDs and the receiver section have photodiode, transimpedance amplifier and analog to digital converter. The LED converts an electrical signal to optical energy that provides illumination as well as communication. Fig. 13 illustrates the principal techniques and the application areas of LiFi technology.

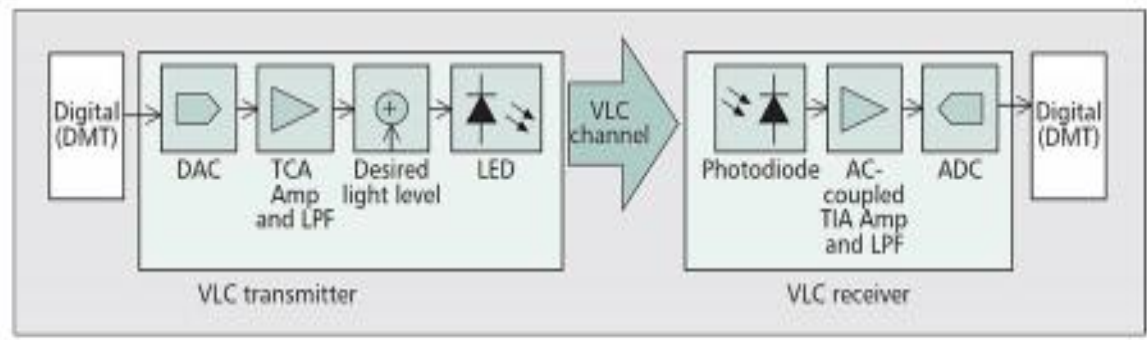

Fig 13. Block diagram of a VLC system [35]

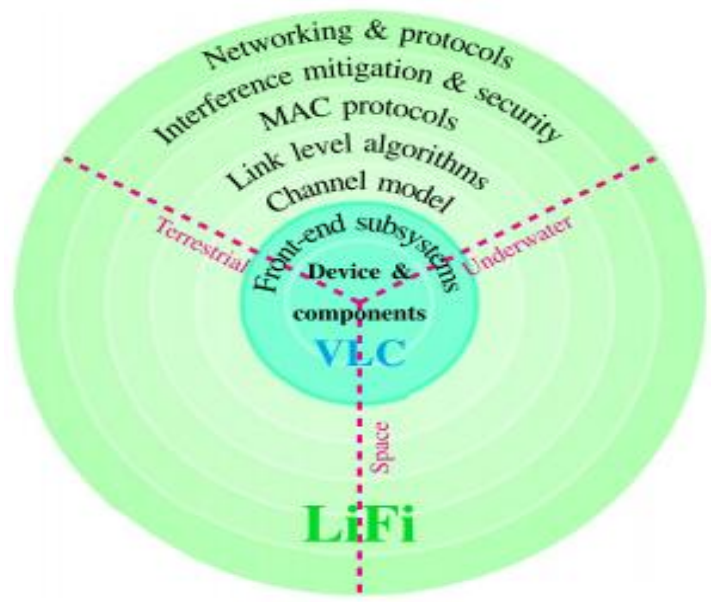

Fig 14 The key elements of LiFi and its application areas [36]

\section{APPLICATIONS OF VLC}

The applications of VLC can be classified in to different areas and in each area, visible light is the mode for communication.

A. Visible Light Communication Based Vehicle Positioning [37]

The vehicle positioning problem has three popular approaches.

\section{Global Positioning System[GPS]}

- Low cost 


\section{International Advanced Research Journal in Science, Engineering and Technology}

Vol. 6, Issue 2, February 2019

- $\quad$ GPS may be unavailable in tunnels or streets in urban areas

- $\quad$ The GPS signals are hindered by high buildings

4G/LTE

- $\quad$ Overcome the limitations of availability of GPS

- $\quad$ Provides tens of meters of positioning accuracy

Light Detection And Ranging[LiDAR]

- $\quad$ Most successful approach

- $\quad$ Provide very high accuracy

- $\quad$ LiDAR demands extra equipment which is generally very

- Expensive

This paper proposes the visible light communication based vehicle positioning system using LED street lights and CMOS image sensors.

\section{B. Distributed Illumination Control [38]}

Indoor illumination control is one of the major application of VLC. New ideas and trends are arising in illumination control within a networked lighting system. Each luminaire consists of a light source, a sensor unit and a communication module. The light source consists of an array of light emitting diodes and it can be dimmed by using a local controller. The sensor unit may be a presence detector to determine the occupant presence and a light sensor for measuring the illuminance. The communication module is used to exchange control information within a local neighborhood. Each luminaire contains a local controller, an LED light source, a light sensor, a presence sensor and a communication module. A photodiode or a light dependent

resistor can be used as a light sensor. Common methods for detecting presence includes passive infrared and ultrasound and the communication can be achieved using IEEE 802.15.7. Each controller have the information about the dimming level of its own luminaire and its closest neighbors.

\section{Connectivity in IoT Indoor Lighting Systems [39]}

An indoor lighting system using Internet of Things[IoT] has been implemented. This system consist of multiple luminaires with IP address, sensors and controllers. These components are able to adapt to their environment. The use of occupancy and light sensors together give a artificial lighting system that can be adapt to user presence and daylight changes. For networked lighting systems, connectivity was assumed in the form of wired interfaces or with wireless radios like ZigBee.

\section{Adaptive Software-Defined Visible Light Communication Networks [40]}

Light bulbs constructed using LEDs connected to the microcontrollers can be used for illumination and communication. The microcontrollers are designed to run software-defined visible light communication[VLC] protocols. Such smart light sources can communicate with each other and with other VLC enabled devices such as smart phones and toys. The LEDs are used for sending and receiving information and there is no need for the photodiodes at the receiver to receive the incoming light signals. The VLC protocol firmware runs on low cost microcontrollers.

\section{E. Digital Image Processing in LED Visible Light Communication [41]}

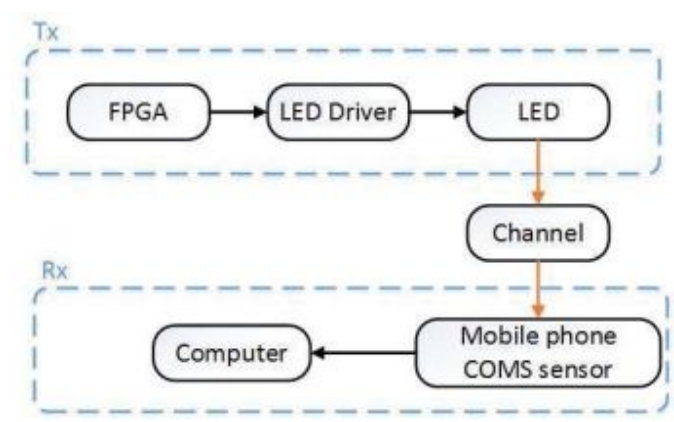

Fig 15. Proposed VLC system [41] 


\title{
International Advanced Research Journal in Science, Engineering and Technology
}

\author{
Vol. 6, Issue 2, February 2019
}

The mobile phone camera can be used as the visible light communication receiver. The camera captures the information in the form of light and dark stripes. These lines can then be decoded by MATLAB tool and the received message is displayed in the computer. In the proposed system, the transmitter is made up of the FPGA, LED driver and LED lamp array with 15 LEDs. An arbitrary binary waveform is generated by FPGA. Then the LED lamp array is lightened up by the LED driver and transmits an On-Off Keying[OOK] signal. The receiver consists of mobile phone CMOS sensor and computer. The modulated white light is received by a mobile phone camera through direct line-ofsight without reflected surfaces.

\section{F. A Non-Invasive Remote Health Monitoring System [42]}

This example came from the need of a safer communication system for health applications. Some of the measuring equipments used in the hospitals give inaccurate reading because of the interference caused by these instruments and operator's personal gadgets like mobile phones. The measuring instruments in hospitals like ECG monitoring system can be connected to the mobile phone using VLC system.

\section{G. Synchronization in Visible Light Communication for Smart Cities [43]}

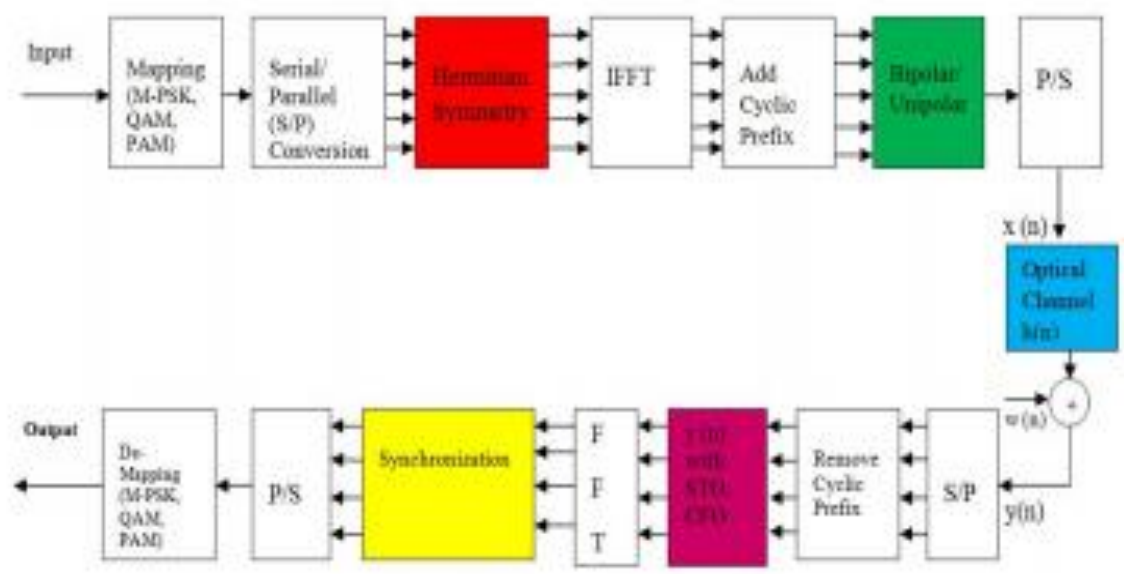

Fig 16. Synchronization in optical OFDM for visible light communication [43]

At the transmitter side, the incoming bit stream is first mapped with the help of modulation formats such

as M-ary Phase Shift Keying[M-PSK], M-ary Quadrature Amplitude Modulation[M-QAM] and M-ary Pulse Amplitude Modulation[M-PAM] respectively. Then this serialized signal is parellelized. Fast Fourier Transform [FFT] module and Inverse Fast Fourier Transform[IFFT] are the other main blocks in the synchronization of optical OFDM.

\section{H. The Uplink Visible Light Communication Beacon System for Universal Traffic Management [44]}

The Universal Traffic Mangement System[UTMS] is a traffic management system beneath the National Police Agency of Japan. Now, 55,000 UTMS infrared beacon systems are installed and they provide express way and ordinary roads information to cars. Now the data rate is $1 \mathrm{Mbps}$ and a faster data rate is necessary to support automotive and smart mobility devices. The future need of the UTMS system, ie the extended bandwidth is obtained by using VLC beacons. In this method, off-the-shelf LED light is used as the transmitter and the photodiode is used as the VLC receiver.

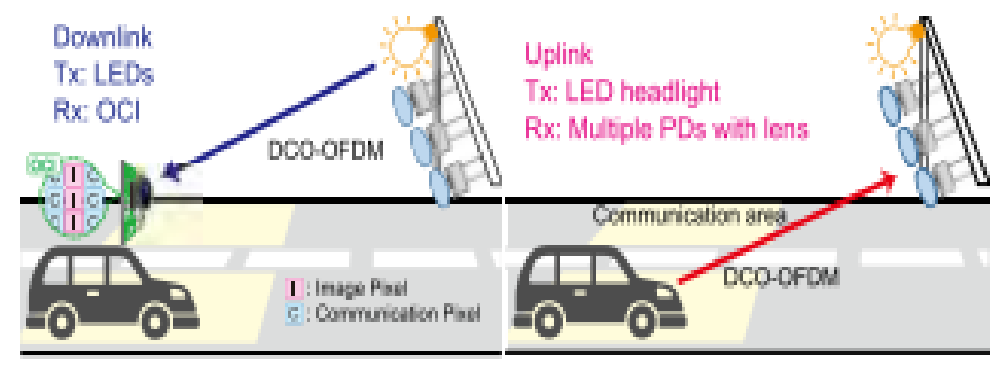

(a)

(b)

Fig 17. Concepts of VLC Beacon System for UTMS (a) Downlink (b) Uplink [44] 


\section{International Advanced Research Journal in Science, Engineering and Technology}

Vol. 6, Issue 2, February 2019

\section{Application Based on Android Smart Device's LED Lamp [45]}

Combining visible light communication with smart devices running on Android system have a wide range of applications. [45] deals with 2 applications including mobile payment and ships communication.

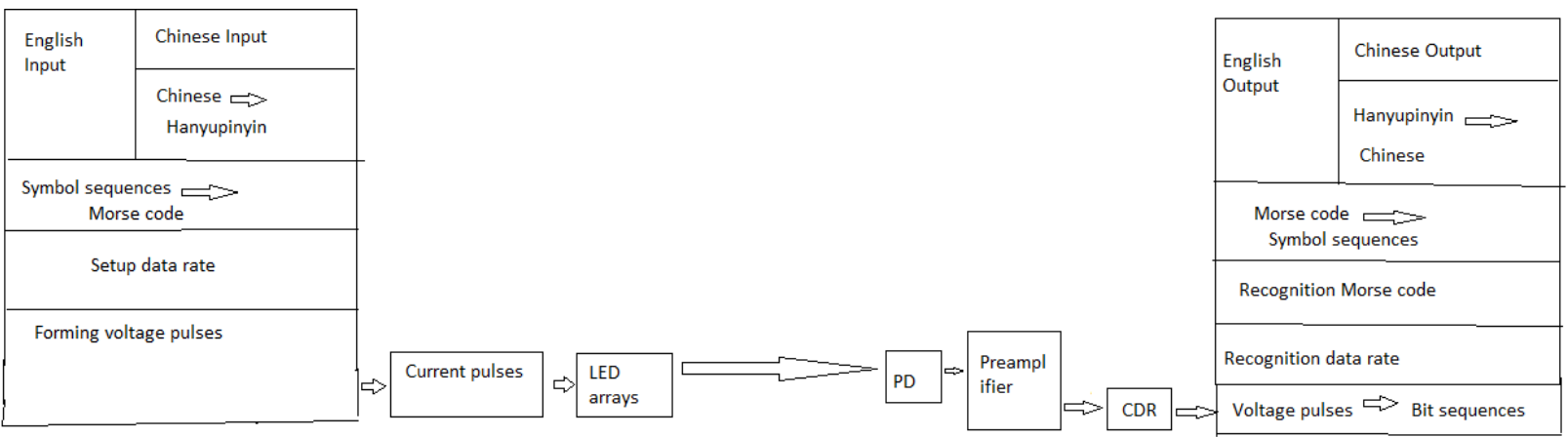

Fig 19. Flowchart of ship communication based on VLC [45]

\section{J. $\quad$ Frame Synchronization of Live Video Streams [46]}

[46] presents the design and implementation of a VLC based video synchronization prototype. The synchronization of different video streams is provided by means of VLC through LEDs and digital cameras. The architecture of the video synchronization system uses visible light as the reference for synchronization. A microcontroller is used to control the light sources and which is placed between the power supply and light sources. The transmitted information is captured by smartphone cameras and streamed to the synchronization servers through network. The sychronization server is placed in a virtualized environment and receives the incoming multimedia streams.

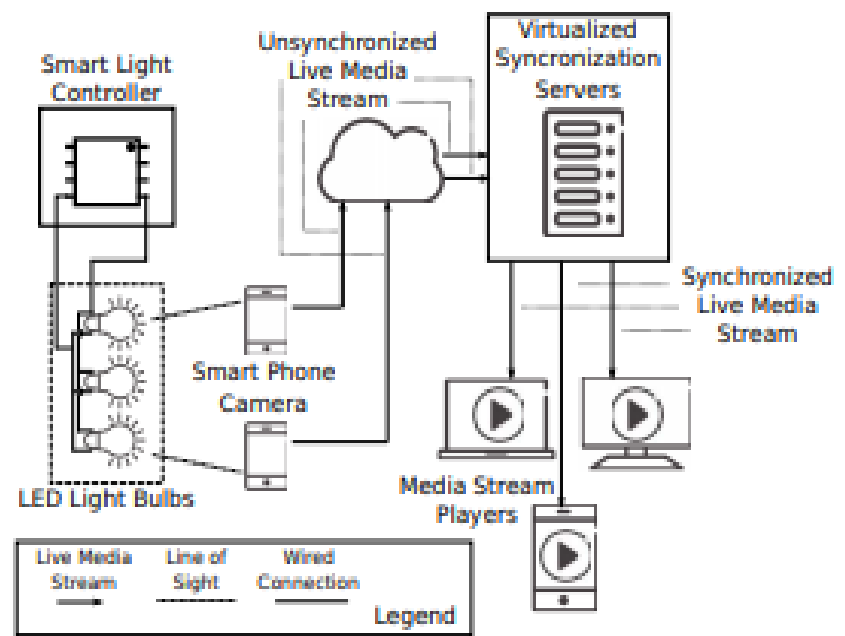

Fig 20. Architecture of the video synchronization system [46]

\section{K. Toys Communicating with LEDs [47]}
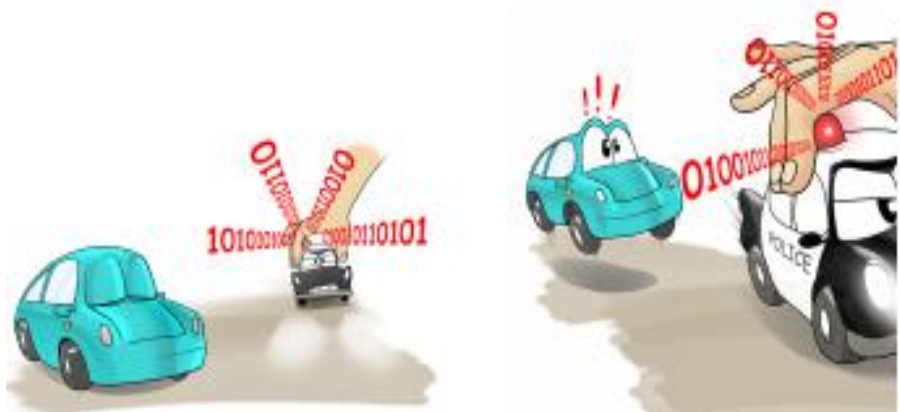

Fig 21.VLC in a smart toy network [47] 


\title{
International Advanced Research Journal in Science, Engineering and Technology
}

\author{
Vol. 6, Issue 2, February 2019
}

Smart toys having communication capabilities are the popular feature now-a-days. [47] deals with a cost effective toy communication using LEDs. In such a method, the messages are sent via VLC are displayed on a screen and it can be passed from one node to another. The toys may react differently to each other depending on how frequently they interact, or how long they have not interacted. The intensity of the LED transmitter can be varied and according to that the data can be communicated wirelessly to a receiver.

\section{Visible Light Technology: A Plethora for Mobile Communications [48]}

The application of white LEDs in the area of visible light communication putforward a low cost data transmission method. There are some areas where the application of visible light is still in its infancy. One such area is the deployment of wireless optical communication in GSM systems. [48] addresses the issues in introducing the concept of frequency breakdown and translation along with the introduction of unique frequency mapping table to accommodate GSM uses into white LEDs. The advantages of circuit simplicity and low power consumption make optical wireless systems favourable for mobile applications. One of the difficulty in connecting GSM systems with visible light technology is the accommodation of $25 \mathrm{MHZ}$ frequency band into the limited modulation bandwidth offered by white LEDs. RoVL[Radio on Visible Light] communication devices consist of visible light transmitters and infrared receivers. The GSM signals are transmitted through LEDs and the infrared is used as the medium. The frequency allocation for these RoVL devices is dependent on the availability of a free channel and the location of the optical wireless cell phone user.

\section{Vehicle to Vehicle Communication using Light Fidelity [49]}

Nowadays, the concepts of road safety and traffic management have importance due to the increased rate of accidents and deaths due to vehicle. Intelligent Transport System[ITS] is the new idea that used to provide various innovative services to facilitate road safety and traffic management. LiFi technology along with LEDs can be used for sending data. The usage of LED eliminates the need of complex wireless networks and protocols. To provide safety on vehicles, a new technique known as VANET is created. The VANET communication is divided into two different categories namely

- $\quad$ Vehicle to Vehicle Communication

- Vehicle to Infrastructure Communication

The first type of communication is between two vehicles[one hop communication]. The Vehicle to Infrastructure Communication is the communication between vehicle and road side infrastructure [multihop communication]. The main objective of this system is to alert drivers when the distance between the two vehicles are reduced below an optimum value. The distance is measured using Ultrasonic sensor.

\section{Indoor Positioning using VLC [50]}

The advantage of using VLC over the traditionally existing indoor positioning technique is that, LED supported indoor localization techniques provide the advantages of high accuracy, no extra equipment for deployment, high security and short response. There are two methods for indoor location estimation using VLC.

- Trilateration

- Angulation

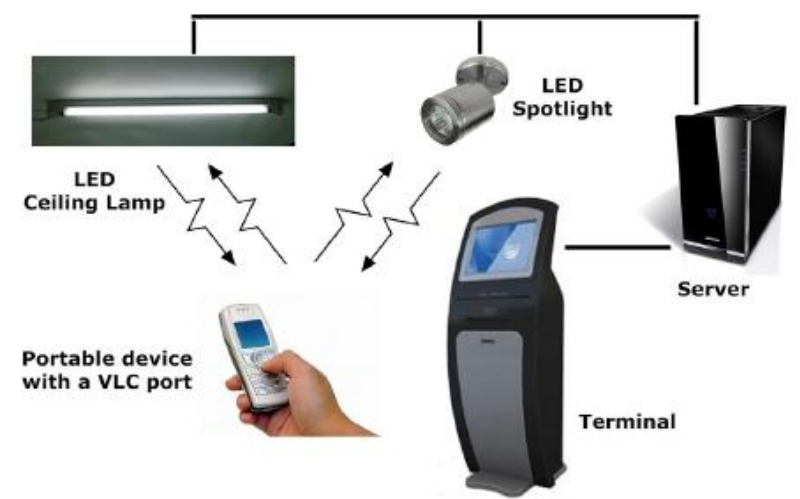

Fig 22. Future broadband wireless networking scenario [51] 


\title{
International Advanced Research Journal in Science, Engineering and Technology
}

\author{
Vol. 6, Issue 2, February 2019
}

In trilateration approach, the target location is estimated by measuring the distances from multiple reference points with known coordinates. The distances can be estimated via different measurements like Received Signal Strength [RSS], Received Signal Strength Ratio[RSSR], Time Difference Of Arrival[TDOA] and distance based positioning. The angulation method estimates the target location by measuring angles from multiple reference nodes. The most popular method for location estimation is to use RSS[50]. A possible scenario of future wireless communication, ie services established through a combination of wired and wireless technologies is given in the figure below.

The wired BSs together with LED based illumination equipment provide wireless network access. Data broadcasting through a ceiling bulb realizes a point-to-multipoint connection and a focused spotlight realizes a point-to-point connection. The Power over Ethernet[PoE] technology can be used to transport data traffic and supply the BSs as well as the lamps with the required power[51].

[52] putforward a typical indoor optical wireless[OW] system.

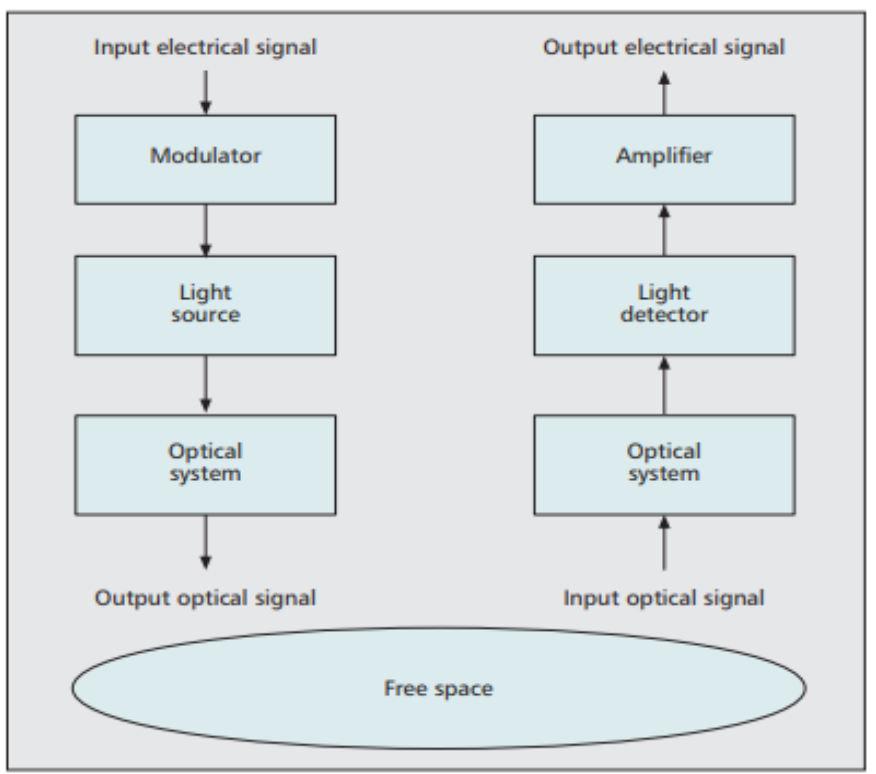

Fig 23. Block diagram of a typical indoor OW system[52]

The basic OW system consists of a light source, free space as the propagation medium and a light detector. The analog or digital signals is given as the input to the electronic circuitry and that modulates the light source. The light source output is passes through an optical system and then in to free space. The received signal passes through the optical system. It may be an optical filter or a lens system or concentrator. The optical filter rejects the optical noise and the lens system or concentrator focuses light on the detector. The output of such an optical system forwarded to a light detector and the resulting photocurrent is amplified. The high data rate requirement of indoor OW systems can be achieved by Multiple Input Multiple Output[MIMO] techniques[52].

The visible light communication based positioning techniques are of two types. ie, photodiodes[PD] and image sensor[IS] based system. Photodiodes are commonly used because they have high sensitivity to light and are also less expensive. The image sensors can spatially separate light sources. The performance metrics for indoor wireless location system includes accuracy, precision and complexity. Accuracy is the average Euclidean distance between the calculated location and the actual location. For a better system, the accuracy must be high. Precision is a measure of the toughness of the positioning process to give the difference in its performance after a number of chances[53].

VLC communication link is designed for both data transmission and illumination. So the already existing LED lighting infrastructure reduces the implementation costs and allow the future expansion of the network. A modified Monte Carlo based ray tracing algorithm[MMCA] is employed to evaluate the VLC channel impulse response for a typical office room as a function of the wavelength, the physical characteristics of the light sources and their layout in the room[54].

The challenges for VLC in vehicle applications includes

- $\quad$ Increasing the robustness to noise

- Increasing the communication range

- $\quad$ Enhancing mobility 


\title{
International Advanced Research Journal in Science, Engineering and Technology
}

\author{
Vol. 6, Issue 2, February 2019
}

- $\quad$ Performing distance measurements and visible light positioning

- Increasing data rate

- $\quad$ Developing parallel VLC

- Developing heterogeneous dedicated short range communications[DSRC] and VLC networks [55]

\section{ADVANTAGES,LIMITATIONS AND APPLICATIONS}

VLC becomes a promising technology due to its advantages. One of the important advantage of VLC includes its fully compatibility to RF communications. The VLC and RF communication methods are complement to each other, form a hybrid or heterogeneous network and further enhancing the communication performances. The VLC technology is safe for the human health. The already existing radiowaves cause cancer in humans and infrared light cause irreversible thermal damage to the cornea. VLC systems do not affect the functionality of the highly sensitive electronic systems and they can be used in RF restricted areas like airplanes, hospitals, chemical and nuclear plants. VLC is also energy efficient since it uses no extra energy for data transmission. The same light can be used for data transmission and illumination. The penetration capabilities of the VLC has both advantages and disadvantages. Since VLC does not penetrate through walls and non transparent obstructions, it provide security to the data being transmitted. The limited penetration capability acts as a disadvantage by limiting the mobility or the coverage area.

\section{A. Advantages}

- It supports larger bandwidth and hence overcome bandwidth limitation of RF communication.

- VLC communication works when both source and receiver are in LOS within the same room. VLC based data communication can not be intercepted by any one from the another room. Hence VLC provides secured communication unlike RF communication.

- VLC source is used for both illumination and communication, it has low power consumption. Hence VLC is power efficient system.

- VLC is light based communication. Hence it is not affected due to EM radiations from RF systems.

- It does not have any health risks to human beings.

- It is easy to install.

\section{B. Limitations}

- VLC based communication has interference issues from other ambient light sources.

- VLC communication supports short coverage range.

- There are challenges to integrate VLC with wifi system.

- Other drawbacks with VLC system are atmospheric absorption, shadowing, beam dispersion etc.

- It requires both source and receiver should be in LOS. Hence non-LOS communication is difficult to be achieved.

\section{Applications}

- Smart lighting: Smart lighting with VLC provide the infrastructure for illumination, control and communications. Such a setup reduce wiring requirements and energy consumption within a building.

- Mobile Connectivity: The communication between two devices can be accomplished by using visible light. Such a communication link have high speed data transmission capabilities with security. The data rate is much higher as compared to Bluetooth or WiFi.

- Hazardous environments: The situations such as mines, petro-chemical plants and oil rigs have the risk of explosions. In such conditions VLC is the safe method for both illumination and communications.

- Vehicle and Transportation: Nowadays the traffic lights and street lights are adopting the LED technology.

- Defence and Security: The visible light can not be detected on the other side of the wall and this advantage has several applications in defence area. The much less penetration capability provide security to the visible light data transmission.

- Hospitals and Healthcare: In scanning equipments and in operation theatres, there is a chance of interference from mobile phone signals. This interference cause inaccurate measurements. Use of visible light as communication medium overcome this limitation.

- WiFi spectrum relief: VLC can provide data rates much higher than the currently existing WiFi and this can be done at low cost. 


\title{
International Advanced Research Journal in Science, Engineering and Technology
}

\author{
Vol. 6, Issue 2, February 2019
}

- Aviation: Radio is undesirable in passenger compartments of aircrafts. LEDs are already used for illumination and can also be used instead of wires to provide media services to passengers. This reduces the aircraft construction costs snd its weight.

- Underwater communications: RF does not work in underwater but visible light can be used for supporting high speed data transmission over short distances in such an environment.

- Location based services: Visible light can be used for identification of different things in a room in quick and accurate manner.

\section{CONCLUSION}

Visible light communication technologies now becoming a advantage in wireless communication scenarios. It's applications spread over a large range of areas and LiFi is the most important VLC technology. The parameters such as capacity, efficiency, availability, security, utilization and safety makes LiFi a promising technology for future applications. The application areas of LiFi includes medical, road safety and traffic management, smart lighting, aviation and underwater communication. The VLC also have large applications, which are on the path of development.

\section{REFERENCES}

[1] Khan, Latif Ullah,'Visible light communication: Applications,architecture,standardization and research challenges",Digital Communications and Networks,vol.3,no.2,pp 78-88,2017,Elsevier.

[2] Kavehrad, M and Amirshahi, P,'Hybrid MV-LV power lines and white light emitting diodes for triple-play broadband access communications", IEC comprehensive report on achieving the triple play: technologies and business models for success,pp 167-178,2006.

[3] Aschenbrenner, N,"500 megabits/second with white LED light",News Release, 18 January,2010.

[4] "St.cloud first to sign on for new technology",(press release),St.Cloud Times,Nov 19,2010.

[5] Harald Haas,"Wireless data from every light bulbs",TEDGlobal,2011.

[6] "LED and visible light communications could be key to unlocking \$5 billion indoor location market",www.abiresearch.com

[7] Haigh, Paul Anthony and Bausi, Francesco and Ghassemlooy, Zabih and Papakonstantinou, Ioannis and Le Minh, Hoa and Fl'echon, Charlotte and Cacialli, Franco,"Visible light communications: real time $10 \mathrm{Mb} / \mathrm{s}$ link with a low bandwidth polymer light-emitting diode",Optics

express,vol.22,no.3,pp. 2830-2838,2014,Optical Society of America

[8] Axrtek MOMO,Axrtek,Inc.

[9] Chowdhury, Mostafa Zaman and Hossan, Md Tanvir and Islam, Amirul and Jang, Yeong Min,"A comparative survey of optical wireless technologies: Architectures and applications",IEEE Access,vol.6,pp. 9819-9840,2018.

[10] Schmid, Stefan and Corbellini, Giorgio and Mangold, Stefan and Gross, Thomas R,"LED-to-LED visible light communication networks",Proceedings of the fourteenth ACM international symposium on Mobile ad hoc networking and computing,pp.1-10,2013.

[11] Ley-Bosch, Carlos and Alonso-Gonzlez, Itziar and Snchez-Rodrguez, David and Ramrez- Casaas, Carlos,"Evaluation of the effects of hidden node problems in IEEE 802.15. 7 uplink performance”, Sensors,vol.16,no.2,pp.216,Multidisciplinary Digital Publishing Institute,2016.

[12] P802.15.7-Standard for short-range wireless optical communication,IEEE,2011.

[13] Wang, Yuanquan and Wang, Yiguang and Chi, Nan and Yu, Jianjun and Shang, Huiliang,'Demonstration of 575-Mb/s downlink and 225-Mb/s uplink bi-directional SCM-WDM visible light communication using RGB LED and phosphorbased LED”,Optics express,vol.21,no.1,pp.1203 $1208,2013$.

[14] Haruyama, Shinichiro,"Visible light communication using sustainable LED lights",2013 Proceedings of ITU Kaleidoscope: Building Sustainable Communities,pp.1-6,2013.

[15] Dietz, Paul and Yerazunis, William and Leigh, Darren,"Very low-cost sensing and communication using bidirectional LEDs",International Conference on Ubiquitous Computing,pp.175-191,2003.

[16] G.Stepnaik,M.Kowalczyk,L.Maksymiuk and J.siuzdak,"Transmission beyond 100Mbit/s using LED both as a transmitter and receiver",IEEE Photonics Technology, October 2015.

[17] Bent, Sarah and Moloney, Aoife and Farrell, Gerald,"LEDs as both optical sources and detectors in bidirectional plastic optical fibre links",2006 IET Irish Signals and Systems Conference,pp.345-349,2006.

[18] Komine, Toshihiko and Nakagawa, Masao,'Integrated system of white LED visible-light communication and power-line communication",IEEE Transactions on Consumer Electronics,vol.49,n0.1,pp.71-79,2003.

[19] Schmid, Stefan and Corbellini, Giorgio and Mangold, Stefan and Gross, Thomas R,'An LED-to-LED Visible Light Communication system with software-based synchronization”,2012 IEEE Globecom Workshops,pp.1264- 1268,2012.

[20] Yang, Zhanyu and Yu, Qianhuan and Zang, Jizhao and Campbell, Joe C and Beling, Andreas,"Phase- Modulated Analog Photonic Link With a High-Power High-Linearity Photodiode",Journal of Lightwave Technology,vol.36,no.18,pp.3805-3814,2018.

[21] Urick, Vincent Jude and Williams, Keith J and McKinney, Jason D,'Fundamentals of microwave photonics”,John Wiley \& Sons, vol.1,2015.

[22] Rajagopal, Sridhar and Roberts, Richard D and Lim, Sang-Kyu,'IEEE 802.15. 7 visible light communication: modulation schemes and dimming support",IEEE Communications Magazine,vol.50,no.3,pp.72-82,2012.

[23] Chen, Chen and Zhong, Wen-De and Yang, Helin and Du, Pengfei,'On the performance of MIMO-NOMA based visible light communication systems",IEEE Photonics Technology Letters,vol.30,no.4,pp.307-310,2018.

[24] Sushma L.Wakchaure,Shailaja D.pawar,Vijay V.Thitme,Bipin B.Shinde,”Overview of LiFi technology”, Amrutvanini polytechnic college,Maharashtra,India,April 2015.

[25] Dinesh Khandal,Sakshi Jain,'LiFi[Light Fidelity]:The future technology in wireless communication",International Journal of Information Computation Technology,ISSN 0974- 2239 Volume 4, Number 16 (2014).

[26] Purohit, Suchit and Tolani, Uma and Goswami, Siddhi,'OnLight Information Broadcasting in Exhibitions Using Li-Fi",Proceedings of 2nd International Conference on Micro-Electronics, Electromagnetics and Telecommunications,pp.135-140,2018.

[27] Kumar, NV Rajeesh and Srikanth, Akella and Singha, Arunangshu and Sam, B Baron,"Comparison of LIFI and WIFI and study of smart meter-survey",2017 International Conference on Information Communication and Embedded Systems (ICICES),pp.1-8,2017.

[28] Roma Jain,Pallavikale,Vidya Kandekar,Pratiksha Kadam,'Wireless data transmission using LiFi technology",IOSR Journal of Electrical and Electronics Engineering (IOSR-JEEE),vol.11,Issue 2 Ver. II,pp.65-68,(Mar.Apr.2016). 


\title{
International Advanced Research Journal in Science, Engineering and Technology
}

\author{
Vol. 6, Issue 2, February 2019
}

[29] Verma, Pushpendra and Shekhar, Jayant and Asthana.A,'Light-Fidelity (Li-Fi): transmission of data through light of future technology" , International Journal of Computer Science and Mobile Computing,vol.4,no.9,pp.113-124,2015.

[30] Kapre, Kshitija Suhas,'Road Traffic Management and Safety Using Li Fi Technology”,International Journal of Advanced Research in Science, Engineering and Technology,vol.2,no.12,2015.

[31] Bhateley,Pooja and Mohindra,Ratul and Balaji,S,"Smart vehicular communication system using Li Fi technology",2016 International Conference on Computation of Power, Energy Information and Commuincation (ICCPEIC),pp.222- 226,2016.

[32] Sindhubala,K and Vijayalakshmi,B,'Design and implementation of visible light communication system in indoor environment”,ARPN Journal of Engineering and Applied sciences,vol.10,no.7,pp.2882-2886,2015.

[33] Yeasmin, Nilufa and Zaman, Rianon and Mouri, Israt Jahan,’Traffic Control Management and Road Safety Using Vehicle to Vehicle Data Transmission Based on Li-Fi Technology”,International Journal of Computer Science, Engineering and Information Technology (IJCSEIT), vol.6, no.3/4,2016.

[34] Arnika Karthikeyan,Aditya K.Iyer,Malvika Kar and C.T.Manimegalai,’Vehicular management using a LiFi communication system powered by BIPV[Building Integrated Photo-Voltaics]",Indian journal of science and technology,vol.10,issue 5,February 2017.

[35] Goswami, Pavas and Shukla, Manoj Kumar,'Design of a li-fi transceiver",Wireless Engineering and Technology,vol.8,no.04,pp.71,2017.

[36] Haas, Harald and Yin, Liang and Wang, Yunlu and Chen, Cheng,"What is lifi?",Journal of Lightwave Technology,vol.34,no.6,pp.15331544,2016

[37] Do, Trong Hop and Yoo, Myungsik,"Visible light communication based vehicle positioning using LED street light and rolling shutter CMOS sensors", Optics Communications,vol.407,pp.112-126,2018.

[38] Caicedo, David and Pandharipande, Ashish,'Distributed illumination control with local sensing and actuation in networked lighting systems", IEEE Sensors Journal,vol.13,no.3,pp.1092-1104,2013.

[39] Warmerdam, Kevin and Pandharipande, Ashish and Caicedo, David,"Connectivity in IoT indoor lighting systems with visible light communications", 2015 ,IEEE Online Conference on Green Communications (OnlineGreenComm),pp.47-52,2015.

[40] Schmid, Stefan and Mangold, Stefan and Gross, Thomas R,’Demo Abstract: Adaptive Software-Defined Visible Light Communication Networks",2017 IEEE/ACM Second International Conference on Internet-of-Things Design and Implementation (IoTDI),pp.273-274,2017.

[41] Li, Zongze and Zhang, Zhenshan and Yuan, Qiaozhi and Qiao, Yaojun and Liao, Ke and Yu, Haihua,'Digital image processing in led visible light communications using mobile phone camera",2016 IEEE International conference on Network Infrastructure and Digital Content (IC NIDC), 239- 243,2016.

[42] Al-Qahtani, Abdulla and Al-hajri, Hamad and Al-kuwari, Saad and Al-yaarabi, Naseer and Al-hababi, Abdulrahman and Al-kubaisi, Essa and Ahmed, Abdulla and Kashef, Mohamad and Abbasi, Qammer H,"A noninvasive remote health monitoring system using visible light communication",2015 2nd International Symposium on Future Information and Communication Technologies for Ubiquitous HealthCare (UbiHealthTech), pp.1-3,2015.

[43] Vappangi, Suseela and Vakamulla, Venkata Mani,"Synchronization in visible light communication for smart cities",IEEE Sensors Journal, vol.18,no.5,pp.1877-1886,2018.

[44] Yamazato, Takaya and Kawagita, Naoaki and Okada, Hiraku and Fujii, Toshiaki and Yendo, Tomohiro and Arai, Shintaro and Kamakura, Koji,"The uplink visible light communication beacon system for universal traffic management",IEEE Access,vol.5,pp.22282-22290,2017.

[45] Wang, Chao and Zhang, Zhidan and Zhang, Minglun and Zhu, Hetian,"Visible light communication application scenarios based on Android smart devices LED lamp",Photonic Network Communications,vol.32,no.2,pp.174-178,2016.

[46] Mehrabi,Maziar and Lafond,S.bastien and Wang,Le,’Frame synchronization of live video streams using visible light communication",2015 IEEE International Symposium on Multimedia (ISM),pp.128-131,2015.

[47] Tippenhauer, Nils Ole and Giustiniano, Domenico and Mangold, Stefan,"Toys communicating with leds: Enabling toy cars interaction”,2012 IEEE Consumer Communications and Networking Conference (CCNC), pp.48-49,2012.

[48] Vijay, Arnesh and Green, Roger J,"Visible Light Technology: A plethora for mobile communications",2012 14th International Conference on Transparent Optical Networks (ICTON),pp.1-4,2012.

[49] Prabhu, TN and Adharsh,M and Ashok,Kumar M and Gokul,Krishna M and Dhayanithi,G,"Vehicle to vehicle communication using light fidelity",International Journal of Computer Applications, vol.164,no.2,2017.

[50] Xu,Wei and Wang,Jia and Shen,Hong and Zhang,Hua and You,Xiaohu,'Indoor positioning for multiphotodiode device using visible-light communications",IEEE Photonics Journal,vol.8,no.1,pp.1-11,2016.

[51] Elgala,Hany and Mesleh,Raed and Haas,Harald,'Indoor broadcasting via white LEDs and OFDM",IEEE Transactions on consumer electronics, vol.55,no.3,pp.1127-1134,2009.

[52] Elgala, Hany and Mesleh, Raed and Haas, Harald,'Indoor optical wireless communication: potential and state-of-the-art",IEEE Communications Magazine,vol.49,no.9,pp.56-62,2011.

[53] Juneja, Sakshi and Vashisth, Sharda,'Indoor positioning system using visible light communication",2017 International Conference on Computing and Communication Technologies for Smart Nation (IC3TSN),pp.79-83,2017.

[54] Ramirez-Aguilera,AM and Luna-Rivera,JM and Perez-Jimenez,R and Rabadan-Borges,J and Guerra,V and Suarez-Rodriguez,C,'Visible light communication constraints in practical indoor lighting systems",pp.1-5,2016.

[55] Cilean,Alin-Mihai and Dimian,Mihai,"Current challenges for visible light communications usage in vehicle applications: A survey",IEEE Communications Surveys\& Tutorials,vol.19,no.4,pp.2681-2703,2017. 